\title{
Forensic Geoscience during CS Investigations and Courtroom Trials without a Murder
}

\author{
Barone $\mathrm{PM}^{* 1,2}$, Di Maggio $\mathrm{RM}^{2}$ and Ferrara $\mathrm{C}^{1,2}$ \\ ${ }^{1}$ Archaeology and Classics Program, the American University of Rome, Rome, Italy \\ ${ }^{2}$ Forensic Geoscience Italy
}

*Corresponding author: Barone PM, Archaeology and Classics Program, The American University of Rome, Rome, Italy, E-mail: p.barone@aur.edu

Citation: Barone PM, Di Maggio RM, Ferrara C (2016) Forensic Geoscience during CS investigations and courtroom trials without a murder. J Forensic Sci Criminol 4(3): 302. doi: doi: 10.15744/2348-9804.4.302

Received Date: May 01, 2016 Accepted Date: June 28, 2016 Published Date: June 29, 2016

\begin{abstract}
Evidence provided by the geosciences can be supportive in a judicial investigation. The forensic geoscientist is able to obtain helpful results from the evidence collected from a crime scene (CS) by combining a variety of analytical methods.

Although the CSI Effect suggests that the TV program and its spin-offs wildly exaggerate and glorify some forensic geosciences, burdening both the prosecution and the defense by creating greater expectations, currently, when the geosciences are involved in real forensic investigations, they are used to search and analyse buried targets following a precise protocol. Nevertheless, recently, some geoscientific approaches to the CS (e.g., geophysics, geoarchaeology, etc.) were relevant to serving justice when dead body was not involved.

As expert witnesses, geoscientists can aid judges by explaining technical matters in easily comprehensible terms. To do this, it is necessary to explain the capability of the geoscientific methods and their applications in 'non-murder' forensic cases.
\end{abstract}

Keywords: Forensic Geoscience; CSI; Forensic Geophysics; GPR; Magnetometer; 'Non-murder' cases

\section{Introduction}

Forensic Geoscience is the application of different branches of Earth Science (e.g. geology, geophysics and geochemistry) to criminal investigation. Analytical techniques developed throughout the $20^{\text {th }}$ century are the base for recent advances in this specific field. In particular, the aim of forensic geosciences is to support the investigation by means of the search and the analysis of the geological evidence. The branches of geoscience that can apply to forensic field are various, for instance, geology, pedology, mineralogy, geomorphology, geoarchaeology, geophysics, geochemistry, statistics, etc. [1-3]. Forensic geoscience techniques can be applied to a wide range of civil law and criminal law issues, relating to such problems as environmental issues, construction failures, pollution and, last but not least, serious crimes such as murder, genocide, terrorism, DVI, drug smuggling and rape [4].

When geoscientific methods are involved in forensic investigations, they are usually used to search for missing bodies, which are generally buried underground, and/or to analyze discovered bodies. In the literature, it is possible to find several studies and examples to this effect $[5,6]$. Recently, these techniques have become relevant for other purposes as well. Geoscientific approaches have become as important in numerous forensic cases 'without a body' as they have been in 'normal' criminal cases, particularly in Italy [7,8]. If the general guidelines followed during the crime scene investigation (CSI) are the same, the differences in the approach are relevant. In Table 1 it is possible to see a typical protocol followed by the forensic geoscientists involved in a crime scene. Note that, in bold it is possible to see the forensic geosciences involved in a 'non-murder' case.

This paper will illustrate two cases in which the forensic geoscience approach was not only necessary but was also important in solving a case. Note that in both in the paper and in the figures, all of the geographical locations have been omitted and are hidden by white rectangles for security and privacy reasons.

The first case involves the Ministry of Cultural Heritage, which places legal restrictions on areas with high archaeological potential. Based on these restrictions, nobody, including the landowners, can remove soil for any purpose, from building new construction to planting. In this case, private landowners can confirm the presence or absence of archaeologically relevant remains beneath their soils. The geophysical method is the best option for this evaluation, and the evidence can be used to ask the Ministry to completely remove or reduce the legal restrictions to a better-defined area. 


\begin{tabular}{|c|c|c|}
\hline General Action & Specific Action & Topic \\
\hline Recovery & $\begin{array}{c}\text { Search } \\
\text { Discovery } \\
\text { Excavation } \\
\text { Stratigraphy (order of events) } \\
\text { Collect evidence }\end{array}$ & $\begin{array}{c}\text { Remote sensing, K9, Geophys- } \\
\text { ics, Archaeology, Geology, } \\
\text { Anthropology, Entomology, } \\
\text { Botany. }\end{array}$ \\
\hline Analyze & $\begin{array}{c}\text { Transfer information } \\
\text { Biological profile } \\
\text { Determine sex, age, and ancestry } \\
\text { Calculate stature } \\
\text { Trauma and pathology } \\
\text { Insects } \\
\text { Vegetal remains } \\
\text { Anthropogenic artifacts }\end{array}$ & $\begin{array}{c}\text { Geology, Anthropology, } \\
\text { Archaeology, Botany, } \\
\text { Entomology }\end{array}$ \\
\hline Identify & Identify activities & Anthropology \\
\hline
\end{tabular}

Table 1: A geoscientific protocol for a crime scene (after [4])

The second case, not strictly related to criminal research, involves the research of pollutants or illegal dumping beneath the soil surface. The chemical and physical modification of the natural soil both in the shallow and in the deep parts of the subsurface creates the perfect dielectric contrast that is detectable by a geophysical tool such as the GPR system.

\section{Materials and Methods}

\section{Case 1}

The geophysical instrument used in the first forensic case is a gradiometer. This specific tool is widely involved in CSI [9,10].

The magnetic gradient survey or gradiometer survey is a passive geophysical tool that detects local variation in the strength of the earth's magnetic field. These variations can be caused by a variety of natural and manmade features that alter the magnetic field emanating from the earth. The difference between the strength of the earth's magnetic field (about 30000 to 60000 nanoteslas [nT]) and the strength of anomalies (typically 1 to $100 \mathrm{nT}$ ) is great, requiring a very sensitive instrument to detect [11].

A gradiometer is an instrument that contains two sensors separated vertically. The top sensor measures the strength of the earth's magnetic field, while the bottom sensor measures the strength of the earth's field as altered by any near-surface anomalies. By subtracting the top measurement from the bottom measurement, the instrument "corrects" for the strength of the earth's magnetic field and "reads" only the local deviation. A gradiometer is ideal for locating small, near surface anomalies, and is therefore very useful in archaeological, geotechnical, environmental, and, of course, forensic mapping. [12].

The gradiometer involved in this case is an Overhauser (GSM-19) instrument by GEM and uses a cycle rate of 0.5 sec, a frequency of approximately $10 \mathrm{~cm}$, a resolution of $0.01 \mathrm{nT}$, and an absolute accuracy of $\pm 0.1 \mathrm{nT}$. The distance between sensors and between profiles is $1 \mathrm{~m}$.

The investigated area was approximately 11 ha on a typical Italian high sand terrace and was entirely covered by the magnetic survey. The quality of all of the raw gradiometer data was high and it was not necessary to apply particular filters or other processing.

\section{Case 2}

The unique ability of Ground Penetrating Radar (GPR) to sense buried artifacts and soil disturbances makes it a powerful tool for police forensic investigations, as shown in several papers [13-15].

A GPR unit essentially produces a pulsed electromagnetic wave that travels through the ground at a velocity controlled by the electromagnetic properties of the investigated material. Differences in relative permittivity (dielectric constant) or electrical conductivity resulting from changes in soil type or groundwater chemistry result in the waves being reflected. The signals reflected from subsurface interfaces or buried/hidden objects are received by the same antenna used for transmission, providing real-time results. GPR operates at frequencies between a few $\mathrm{MHz}$ and $3 \mathrm{GHz}$, and the depth of the penetration is sensitive to the electrical properties of the ground.

To correctly interpret a radargram, it is necessary to know how the section was derived. The transmitted pulse from the radar antenna does not travel vertically downward but spreads out in a cone, which indicates that the isolated target is "seen" before the antenna is directly over it. This cone of transmission enlarges with increasing depth below the surface. Subsurface conditions and the transmitted frequency into the ground determine the dimensions of this cone, and higher frequencies produce narrower cones [16].

The system used in this second forensic case is the GPR FINDAR (Sensors \& Software, Inc.), equipped with $500 \mathrm{MHz}$ bistatic and non-separable antennas with an odometer. The area (a total of $3500 \mathrm{sqm}$ ) is investigated with several singular profiles. A grid with 
dimensions of $10 \times 10 \mathrm{~m}$ for collecting parallel profiles with a line spacing of $0.5 \mathrm{~m}$ is acquired by following only the $\mathrm{Y}$ orientation, a stacking of 4 and a step-size of $0.025 \mathrm{~m}$. Finally, a signal velocity of $0.08 \mathrm{~m} / \mathrm{ns}$ was calculated. Note that, to estimate the depth of a target, some information on the pulse velocity in the subsurface should be retrieved, using simple calibration methods or more refined signal inversion techniques [17].

\section{Results}

\section{Case 1}

In the first case, the private landowners of this area had the opportunity to confirm the presence of archaeologically relevant remains beneath their soils using GPR and to ask the Superintendence and the Ministry to remove or reduce the legal restriction to a better defined area.

In particular, the archaeological find consists of a series of post holes associated with huts, clay fragments from ceramic dishes, some botanical remains, and remains of decorative elements dating back to protohistory. The discovery has been widely documented and is almost entirely stored in the local museum, excluding the largest wall structures [7]. In this regard, it was necessary to perform a careful analysis to determine how the relevant portion of land had been excavated and how extensive was the archaeological finding in relation to the cadastral excerpt present in the report of the Superintendence.

From 1989 to 2007, the research was focused mainly on a certain part of the sandy plateau. In particular, just three cadastral parcels were investigated by archaeology, for a total of approximately 2 ha.

After 2007, when the temporary expropriation made by the local Archaeological Superintendence was finished, the excavations were backfilled, and the most relevant remains were collected [7]. In 2014, the local Superintendence together with the Ministry of Cultural Heritage decided to place more severe legal restrictions on the area. The restrictions do not allow building or cultivating or, generally, the removal of soil from the area of interest.

The magnetic survey demonstrates how the areal dimensions of the Superintendence restriction were objectively too large. The area affected by this geophysical prospection was approximately 11 ha and demonstrated how the use of this geophysical tool has allowed for both high resolution and detailed results. In fact, on the magnetic maps the gradiometer shows a strong anomaly in the subsoil related to the remains previously excavated and documented by the local Superintendence. The depth is probably in accordance to the same depth of the archaeological remains, between $0.10 \mathrm{~m}$ and $0.50 \mathrm{~m}$ (Figure 1).

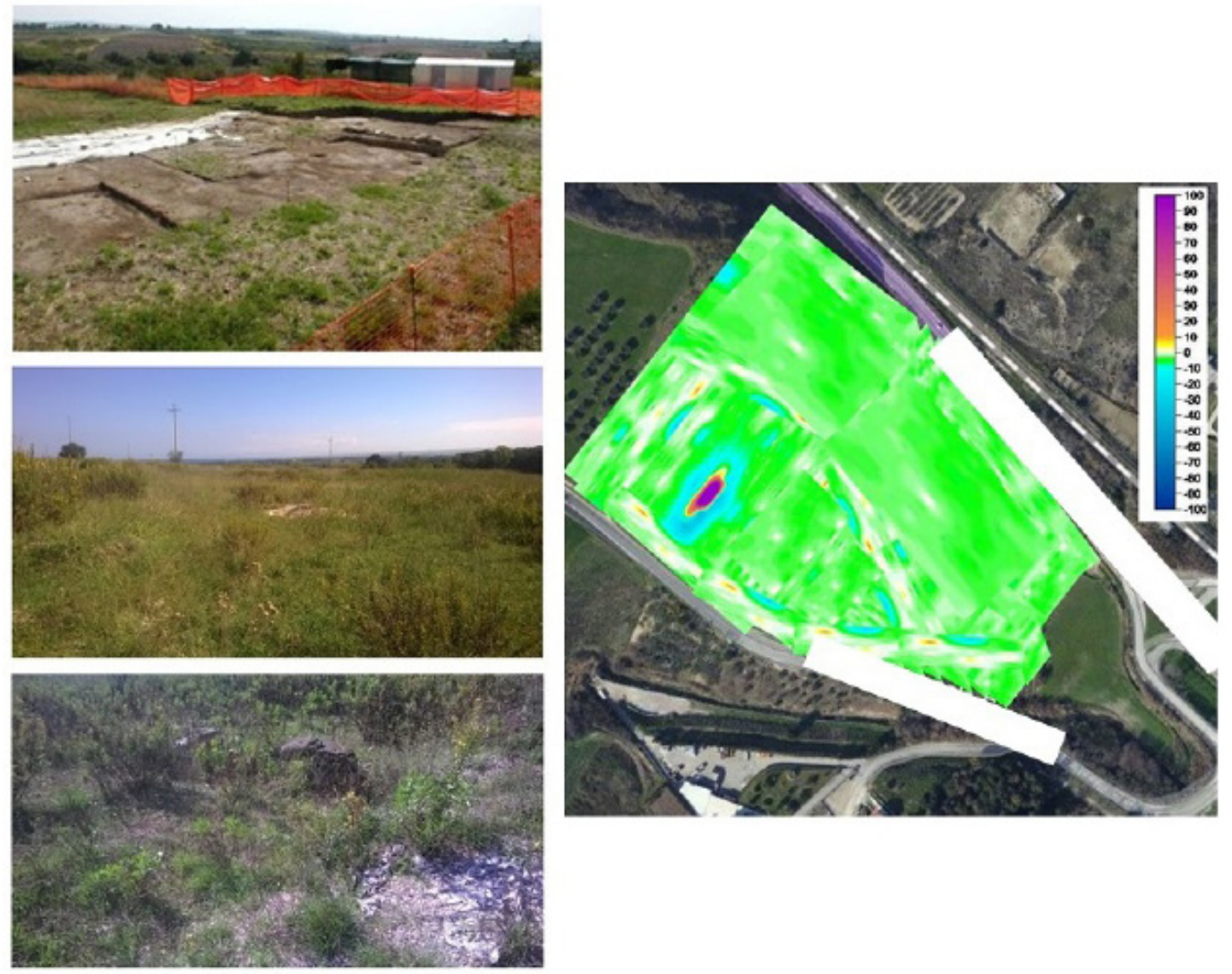

Figure 1: In the figure it is possible to see, on the left, the evolution of the archaeological excavations from 1998 until today. On the right, the result of the magnetic survey in the whole area (11 ha) in which the anomaly related to the previous archaeological remains is well visible (approximately 2 ha). Note that the yellow anomalies around the main one are due to a rural pathway 
The gradiometer confirms the archaeological presence in only a limited area ( $2 \mathrm{ha}$ ) and probably not deeper than $1 \mathrm{~m}$. Moreover, this geophysical survey highlights the complete absence of other remains beneath the soil.

\section{Case 2}

The second case illustrates the GPR analysis close to a very popular beach. This investigation is currently underway.

Based on the police hypothesis, the area under forensic investigation suffered from the illegal burial of industrial muds from a water purifier on the shore. The aim of the survey was to find this burial site in a specific private field. Single GPR profiles were acquired inside this area, in an attempt to find anomalies in the subsoil. Based on one of these radargrams (Figure 2), a more detailed grid was acquired as specified in the paragraph above.

Both the radargram and the depth-slice clearly show the presence of a well-defined layer at a depth of $2.15 \mathrm{~m}$. Based on its shape and depth, this anomaly could be related to the presence of the illegally dumped muds (Figure 2). Chemical analyses of this layer are ongoing to confirm such results.
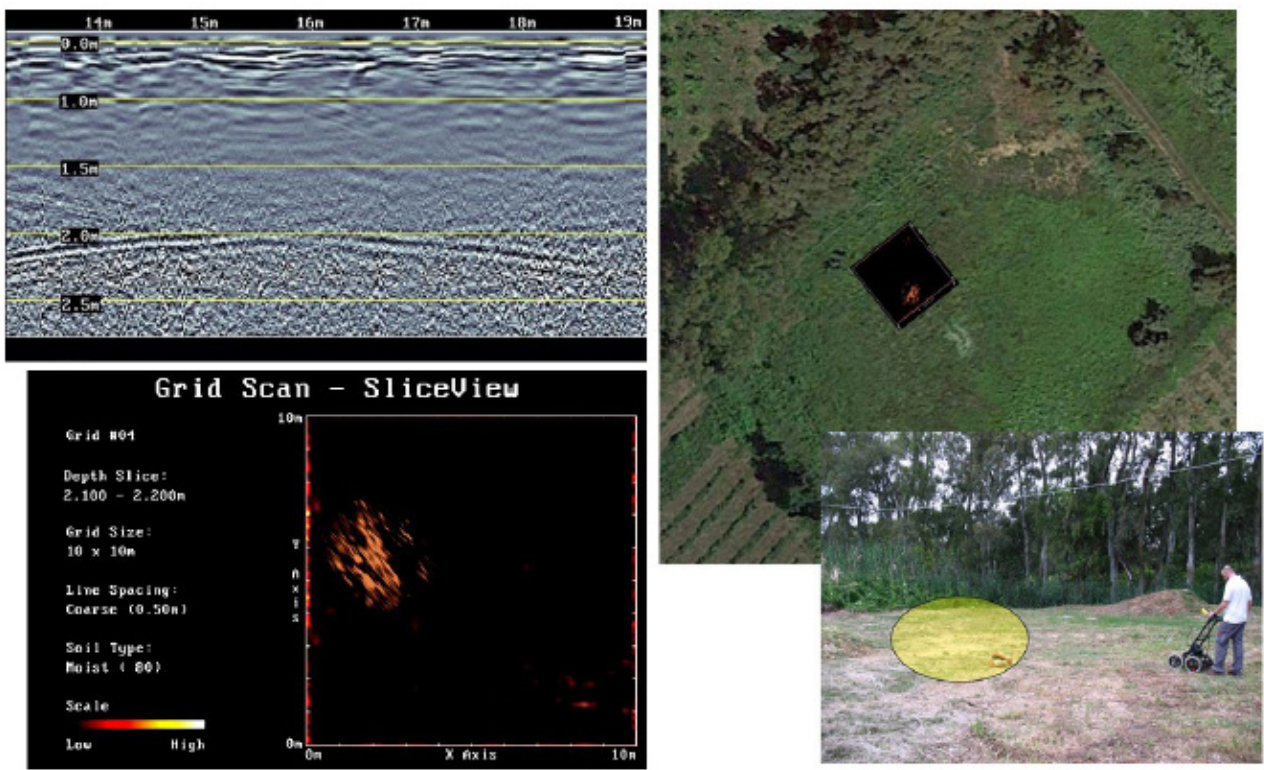

Figure 2: On the left, the GPR anomaly is very visible at the same depth $(2.15 \mathrm{~m})$ in both the radargram (top left) and the depth-slice (bottom left). On the right, the overlap of the GPR map (top right) and the location of the anomaly on the surface (bottom right)

\section{Conclusion}

Beyond the traditional forensic fields, a broad range of science and expertise is used for forensic purposes. The two case studies illustrated in this paper show that the successful application of geoscience in forensic analysis could also be helpful during CSI not involving a body search or examination. The results showed the various possibilities in which forensic geophysical investigations can help to solve a 'non-murder' case at the same level of high standards and with the same distinguished results of a 'murder' case.

In particular, in the first case, the GPR and gradiometer survey scientifically refuted the possibility of finding further archaeological remains in the investigated area and reset the level of archaeological potential in the area, giving the Ministry of Cultural Heritage and its Superintendence a unique possibility to better define the restrictions.

Moreover, in the second case, the GPR technique allowed definition of the subsoil the presence of pollution material covered by natural sand in a popular public beach.

Based on these results, geophysical methods demonstrate the capability to aid law enforcement in the research and recovery of also these specific targets because they can be used to investigate large areas non-destructively and rapidly where a suspect, illegal burial or, in general, a forensic target was hidden in the subsoil.

In general, forensic geoscience can be most effective in a criminal investigation in which the geoscientist is regarded as part of the investigative team early in the case. If the geoscientist is provided appropriate information about the case history and the dynamics of the crime, the analyses can effectively maximize the possibility of solving a case.

\section{References}

1. Morgan RM, Bull PA (2007) Forensic Geoscience and crime detection. Minerva Med Leg 127: 73-89.

2. Ruffell A, McKinley J (2005) Forensic geoscience: applications of geology, geomorphology and geophysics to criminal investigations, Earth Sci Rev 69: 235-47.

3. Bergslien E (2012) An introduction to Forensic Geoscience. Wiley-Blackwell ISBN: 978-1-4051-6054-4. 
4. Pye K, Croft DJ (2004) Forensic Geoscience: introduction and overview. The Geological Society of London 232: 1-5.

5. Barone PM, Di Maggio RM, Ferrara C (2015) Forensic Geo-Archaeology in Italy: Materials for a Standardisation. Int J Archaeol Sci 3: 45-56.

6. Di Maggio RM, Barone PM, Pettinelli E, Mattei E, Lauro SE, et al. (2013) Geologia Forense. Introduzione alle geoscienze applicate alle indagini giudiziarie. Flaccovio Editore.

7. Barone PM, Di Maggio RM, Ferrara C (2015) Not necessarily buried bodies: forensic GPR investigations from criminal to civil justice. Proc $8^{\text {th }}$ Int Workshop Adv Ground Penetrating Radar IEEE, Florence 1-4.

8. Murphy BL, Morrison LD (2014) Introduction to Environmental Forensics. Academic Press Elsevier.

9. Novo A, Lorenzo H, Rial FI, Solla M (2011) 3D GPR in forensics: Finding a clandestine grave in a mountainous environment. Forensic Sci Int 204: 134-8.

10. Pringle JK, Jervis J, Cassella JP, Cassidy NJ (2008) Time-Lapse Geophysical Investigations over a Simulated Urban Clandestine Grave. J Forensic Sci 53: $1405-16$.

11. Tabbagh J (2003) Total field magnetic prospection: are vertical gradiometer measurements preferable to single sensor survey? Archaeol Prospect 10: 75-81.

12. Davenport GC (2001) Remote sensing applications in forensic investigations. Hist Archaeol 35: 87-100.

13. Ruffell A, McKinley J (2008) Geoforensics. John Wiley and Sons Ltd., UK.

14. De Souza T (2009) Ground penetrating radar for forensic. CINDE J 30: 16-7.

15. Barone PM, Ferrara C, Pettinelli E, Annan AP, Fazzari A, et al. (2012) Forensic Geophysics: how GPR could help police investigations. Proc 18 ${ }^{\text {th }}$ Eur Meeting Environ Engineer Geophys Near Surface Geosci Divi EAGE.

16. Jol H (2009) Ground Penetrating Radar Theory and Applications. Elsevier.

17. Pettinelli E, Barone PM, Di Matteo A, Mattei E, Lauro SE (2011) Radio waves technique for non-destructive archaeological investigations. Contemp Phys 52: 121-30.

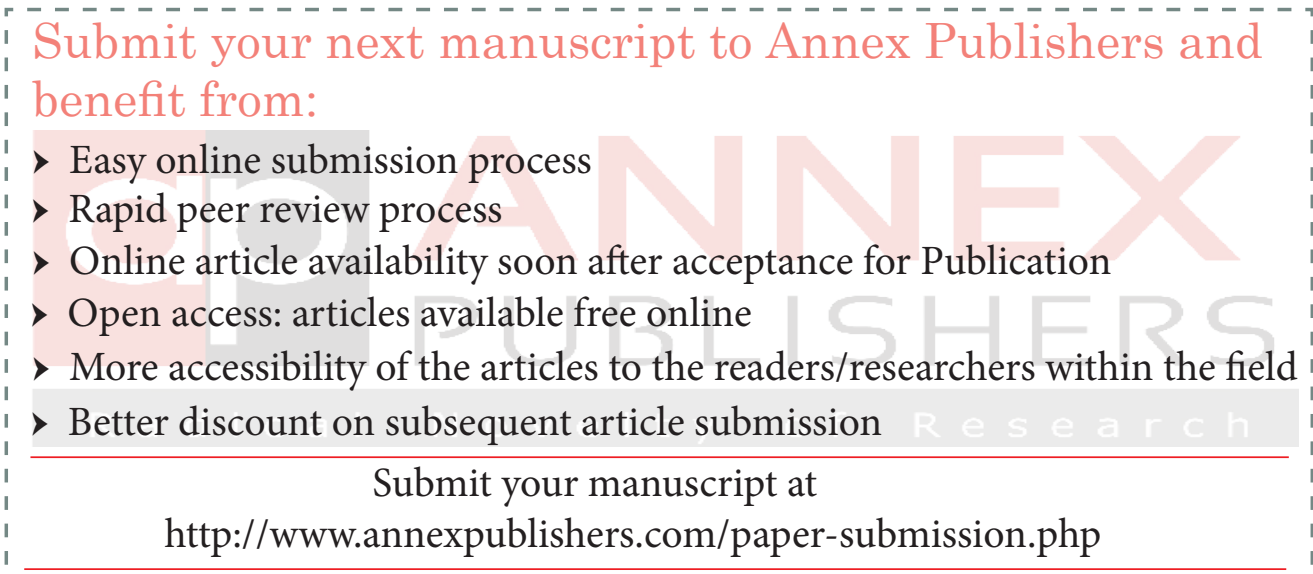

